\title{
THE DERIVATIVE OF A HOLOMORPHIC FUNCTION AND ESTIMATES OF THE POINCARÉ DENSITY
}

\author{
By SHINJi YAMASHITA
}

\begin{abstract}
Let $P_{G}(z)$ be the Poincaré density of the Poincaré metric $P_{G}(z)|d z|$ in a domain $G$ in the complex plane $C$ such that $C \backslash G$ contains at least two points. Let $\boldsymbol{\delta}_{G}(z)$ be the distance of $z \in G$ and the boundary of $G$ in $\boldsymbol{C}$. It is well known that $\delta_{G}(z) P_{G}(z) \leqq 1$ everywhere, and if $G$ is simply connected further, then $1 / 4 \leqq \delta_{G}(z) P_{G}(z)$ everywhere. These inequalities have their roots in the classical and general inequalities of A. J. Macintyre, W. Seidel and J.L. Walsh for holomorphic functions defined in the open unit disk $D$. We prove sharp inequalities for the derivative of a holomorphic function in $D$, inequalities which, in particular, generalize the classical ones. Applications to $P_{G}$ will be given.
\end{abstract}

1. Introduction and some results. We shall prove some sharp inequalities for the Bloch derivative

$$
F(z, f)=\left(1-|z|^{2}\right)\left|f^{\prime}(z)\right|
$$

of a function $f$ holomorphic in $D=\{|z|<1\}$. To show how our results are effective, even in somewhat restricted expression, we remember here the classical results due to A. J. Macintyre [Mc] and W. Seidel and J.L. Walsh [SW]. Let $\delta(z, f)$ be the maximum of $r>0$ such that the Riemann image surface of $D$ by $f$ contains the one-sheeted disk $\{w ;|w-f(z)|<r\}$ of center $f(z)$. We set $\delta(z, f)=0$ if $f^{\prime}(z)=0$. It is easy to observe that $\delta(z, f)<+\infty$.

The following are known:

$$
\delta(z, f) \leqq F(z, f), \quad z \in D .
$$

(II) If $f$ is univalent in $D$ further, then

$$
F(z, f) / 4 \leqq \delta(z, f), \quad z \in D .
$$

See $\left[\mathbf{S W}\right.$, p. 133, Theorems 2 and $\left.1^{\prime}\right]$. Note that (II) is essentially due to

1980 Mathematıcs Subject Classificatıon (1985 Revisıon). Primary 30D99; Secondary $30 \mathrm{C} 20,30 \mathrm{C} 50,30 \mathrm{C} 55,30 \mathrm{D} 45$.

Received May 21, 1991. 
Macintyre [Mc, Theorem 1]. See Remark 1 at the end of Section 5 in the present paper also. Set

$$
\gamma(z, f)=\left(1-|z|^{2}\right)|(\partial / \partial z) \log F(z, f)|, \quad z \in D,
$$

where $2 \partial / \partial z=\partial / \partial x-i \partial / \partial y$ for $z=x+i y$. If $f^{\prime}(z)=0$, then we set $\gamma(z, f)=+\infty$. Note that $\gamma(z, f) \leqq 2$ if $f$ is univalent in $D$ (see, for example, [G, I, p. 63, (5)]). As will be seen, $\gamma(z, f)$ has some important meanings. For $z \in D$ we let $\mathscr{M}_{z}$ be the set of Möbius transformations $T(w)=\alpha(w-z) /(1-\bar{z} w),|\alpha|=1$. We have the following improvements of (I ) and (II).

$$
\delta(z, f) \leqq \frac{2 F(z, f)}{2+\gamma(z, f)}, \quad z \in D .
$$

The equality at $z$ with $f^{\prime}(z) \neq 0$ holds if and only if

$$
f=p T+q,
$$

where $T \in \mathscr{M}_{z}$ and $p \neq 0$ and $q$ are complex constants.

(II') If $f$ is univalent in $D$ further, then

$$
\frac{F(z, f)}{2+\gamma(z, f)} \leqq \delta(z, f), \quad z \in D \text {. }
$$

The equality at $z$ holds if and only if

$$
f=\frac{p k \cdot T}{1+R k \circ T}+q
$$

where (a) $T \in \mathscr{M}_{z}$; (b) $k \circ T$ is the composed function, first $T$ and then the Koebe function $k(w)=w /(1-w)^{2}$; (c) $p \neq 0$ and $q$ are complex constants ; (d) $R$ is a constant, $0 \leqq R \leqq 4$. The function $f$ of (1.2) maps $D$ one-to-one onto the complex plane $C$ slit along the half lines:

$$
\begin{aligned}
& \left\{p t+q ; \frac{1}{R} \leqq t<+\infty\right\} \quad(=\emptyset, \text { the empty set, if } R=0), \\
& \left\{p t+q ;-\infty<t \leqq \frac{1}{R-4}\right\} \quad(=\emptyset, \text { if } R=4) .
\end{aligned}
$$

We remember that (I) and (II) have applications to the estimates of the Poincaré density $P_{G}$. A domain $G \subset C$ whose complement $C \backslash G$ contains at least two points has the Poincaré metric $P_{G}(z)|d z|, z \in G$. More precisely, for a holomorphic, universal covering projection $f$ from $D$ onto $G$, or $f \in$ $\operatorname{Proj}(D, G)$ in notation, we have the expression

$$
1 / P_{G}(z)=F(w, f), \quad z=f(w), w \in D .
$$

The right-hand side is independent of the particular choice of $f$ and $w$ as far as $z=f(w)$. The density $P_{G}$ is then a $C^{\infty}$ function in $G$, and we can show [Y, p. $167,(3.2)]$ that 


$$
\gamma_{G}(z) \equiv\left|(\partial / \partial z)\left\{1 / P_{G}(z)\right\}\right|=\gamma(w, f), \quad z \in G,
$$

where $z=f(w), f \in \operatorname{Proj}(D, G)$. Let

$$
\delta_{G}(z)=\inf _{w \in \partial G}|w-z|, \quad z \in G,
$$

where $\partial G$ is the boundary of $G$ in $C$. We note that

$$
\delta_{G}(z)=\delta(w, f), \quad \text { for } z=f(w), f \in \operatorname{Proj}(D, G), w \in D .
$$

The following are consequences of (I ) and (II):

$$
\delta_{G}(z) P_{G}(z) \leqq 1, \quad z \in G .
$$

(IIP) If $G$ is simply connected, then

$$
1 / 4 \leqq \delta_{G}(z) P_{G}(z), \quad z \in G .
$$

Note that there is no reference to the Poincare density in both papers [Mc, $\mathbf{S w}$ ]. Also no reference to [Mc, Sw] is made in [K, p. 43 et ff.]. The next two propositions follow from $\left(\mathrm{I}^{\prime}\right)$ and $\left(\mathrm{II}^{\prime}\right)$ :

$$
\delta_{G}(z) P_{G}(z) \leqq \frac{2}{2+\gamma_{G}(z)}(\leqq 1), \quad z \in G .
$$

The equality holds at (only) one point $z \in G$ if and only if $G$ is a disk and $z$ is its center.

(II'P) If $G$ is simply connected, then

$$
(1 / 4 \leqq) \frac{1}{2+\gamma_{G}(z)} \leqq \delta_{G}(z) P_{G}(z), \quad z \in G .
$$

The equality holds at (only) one point $z \in G$ if and only if $G$ is $C$ slit along the half lines (1.3) and (1.4) and $z=q$.

Throughout the present paper Univalent Function Theory occupies fundamental parts. As main references we adopt the books [G] of A.W. Goodman.

Makoto Masumoto and Nobuyuki Suita helped the author to obtain copies of the papers of Fenchel and Pick. M. Masumoto, Yoshihiro Mizuta, and Masakazu Shiba attended the author's seminar on almost all the subjects in the present paper, at Hiroshima University during March 12-14, 1991, and gave him many invaluable criticisms. Without their assistance he would have spent much time to complete the present paper. It is a pleasant duty for the author to express his cordial thanks to all of them.

2. Some preliminary notes. For $f$ holomorphic in $D$, we let $\rho(z, f), z \in D$, be the maximum of $r, 0<r \leqq 1$, such that $f$ is univalent in the Apollonius disk $\{w ;|w-z| /|1-\bar{z} w|<r\}$. If $f^{\prime}(z)=0$, then we define $\rho(z, f)=0$. If $f^{\prime}(z) \neq 0$, then we have 


$$
\frac{f\left(\frac{w+z}{1+\bar{z} w}\right)-f(z)}{\left(1-|z|^{2}\right) f^{\prime}(z)}=w+a(z, f) w^{2}+\cdots, \quad w \in D
$$

where

$$
a(z, f)=\frac{1}{2}\left(1-|z|^{2}\right) \frac{f^{\prime \prime}(z)}{f^{\prime}(z)}-\bar{z}=\left(1-|z|^{2}\right)(\partial / \partial z) \log F(z, f) .
$$

Thus, $\gamma(z, f)=|a(z, f)|$. We begin with

LEMMA 2.1. If $f^{\prime}(z) \neq 0$ at $z \in D$ for $f$ holomorphic in $D$, then

$$
\rho(z, f) \gamma(z, f) \leqq 2 .
$$

The equality in (2.1) holds if and only if $f$ is of the form

$$
f=p k \circ T+q,
$$

where $T \in \mathscr{M}_{z}$ and $p \neq 0$ and $q$ are complex constants; this is the case $R=0$ in (1.2).

Proof. Let $S$ be the family of functions $h$ holomorphic and univalent in $D$ with $h(0)=h^{\prime}(0)-1=0$. Set $\rho=\rho(z, f)(>0)$. Then the function of $w \in D$,

$$
g(w)=\frac{f\left(\frac{\rho w+z}{1+\bar{z} \rho w}\right)-f(z)}{\rho\left(1-|z|^{2}\right) f^{\prime}(z)}=w+\rho a(z, f) w^{2}+\cdots, \quad w \in D,
$$

is in $S$. The estimate (2.1) is just the Bieberbach second coefficient theorem [G, I, p. 33, Theorem 1] for $g$. It is not difficult to see that the equality holds for $f$ of (2.2). Conversely suppose that the equality in (2.1) holds. Then, $g=k_{\alpha}$, where $k_{\alpha}$ is a rotation of the Koebe function $k$, that is, $k_{\alpha}(w)=w /(1-\alpha w)^{2}$, $w \in D,|\alpha|=1$. We thus have

$$
f(w)=p^{\prime} k_{\alpha}\left(\frac{\rho^{-1}(w-z)}{1-\bar{z} w}\right)+q,
$$

where $p^{\prime}=\rho\left(1-|z|^{2}\right) f^{\prime}(z)$ and $q=f(z)$. If $\rho<1$, then $f$ must have a pole at $\left(\rho \alpha^{-1}+z\right) /\left(1+\bar{z} \rho \alpha^{-1}\right) \in D$. This contradiction shows that $\rho=1$, and hence we have (2.2) with $p=p^{\prime} \alpha^{-1}$ and $T(w)=\alpha(w-z) /(1-\bar{z} w)$.

We end this section by proving the continuity of $\delta(z, f)$ and $\rho(z, f)$ as functions of $z \in D$ though this fact will not be made use of until in Remark 2 in Section 5. First, given $z \in D$ we can find $\varepsilon \equiv \varepsilon(z)>0$ such that

$$
|\delta(z, f)-\delta(w, f)| \leqq|f(z)-f(w)| \quad \text { for }|z-w|<\varepsilon .
$$

If $f^{\prime}(z)=0$, then this is obvious because $\delta(w, f)=|f(z)-f(w)|$ for small $|z-w|$. In case $f^{\prime}(z) \neq 0$, we can choose $\varepsilon>0$ such that, if $|z-w|<\varepsilon$, then $f(w)$ is in the one-sheeted disk of center $f(z)$ and radius $\delta(z, f) / 2$ on the Riemann image surface. Then, 


$$
\delta(z, f)-|f(z)-f(w)| \leqq \delta(w, f) \text { and } \delta(w, f)-|f(z)-f(w)| \leqq \delta(z, f),
$$

which show (2.4). We next prove that

$$
|\rho(z, f)-\rho(w, f)| \leqq|z-w| /|1-\bar{z} w| \quad \text { in } D .
$$

We note that $|z-w| /|1-\bar{z} w|$ satisfies the triangle inequality, so that this is a metric in $D$. In case $f^{\prime}(z)=0$ or $f^{\prime}(w)=0,(2.5)$ is obvious. In case $f^{\prime}(z) \neq 0 \neq$ $f^{\prime}(w)$ we may suppose that $\rho(z, f)>\rho(w, f)$ by the symmetry of (2.5) in $z$ and $w$. If $|z-w| /|1-\bar{z} w| \geqq \rho(z, f)$ we have nothing to prove. If $|z-w| /|1-\bar{z} w|$ $<\rho(z, f)$, then $\rho(z, f)-|z-w| /|1-\bar{z} w| \leqq \rho(w, f)$; this is (2.5).

3. An upper estimate. We consider $\rho, \gamma$, and $\delta$ in

THEOREM 1. Let $f$ be holomorphic in $D$ and suppose that $f^{\prime}(z) \neq 0$ at a point $z \in D$. Then,

$$
\frac{\rho(z, f) F(z, f)}{2+\rho(z, f) \gamma(z, f)} \leqq \delta(z, f) .
$$

The equality holds if and only if $f$ is of the form (1.2).

For the proof of Theorem 1 we make use of

LEMMA 3.1. If $f \in S$, then

$$
\frac{2}{4+\left|f^{\prime \prime}(0)\right|} \leqq \delta(0, f) .
$$

The equality holds if and only if

$$
f=\frac{k_{\alpha}}{1+R \alpha k_{\alpha}}
$$

for $|\alpha|=1$ and $0 \leqq R \leqq 4$.

Goodman attributes (3.2) to W. Fenchel [G, II, p. 245, Theorem 33] and Fenchel attributes (3.2) "zum Beispiel" to G. Pólya and G. Szegö; see the new English edition [PS] (in particular, p. 24, Problem 148, and its answer in p. 195) of the Pólya-Szegö problem book cited in [F, p. 431]. The equality discussion in our form is never given in the cited literatures, so that it must be completed.

Let $c \in C \backslash f(D)$ and set

$$
g(z)=\frac{c f(z)}{c-f(z)}=z+\left(\frac{1}{c}+\frac{f^{\prime \prime}(0)}{2}\right) z^{2}+\cdots, \quad z \in D .
$$

Since $g \in S$ it follows that

$$
\frac{1}{|c|}-\frac{\left|f^{\prime \prime}(0)\right|}{2} \leqq\left|\frac{1}{c}+\frac{f^{\prime \prime}(0)}{2}\right| \leqq 2 .
$$


Hence

$$
|c| \geqq \frac{2}{4+\left|f^{\prime \prime}(0)\right|}
$$

We therefore have (3.2). For $f$ of (3.3) we have $\left|f^{\prime \prime}(0)\right|=2|R-2|$ and

$$
\delta(0, f)=\left\{\begin{array}{cl}
\frac{1}{4-R} & \text { if } R \leqq 2 \\
\frac{1}{R} & \text { if } R>2 .
\end{array}\right.
$$

We thus have the equality in (3.2). Conversely suppose that the equality in (3.2) holds. We then choose $c$ on the boundary $\partial f(D)$ such that $|c|=\delta(0, f)$. Thus, it follows from the one-quarter theorem of P. Koebe (see [G, I, p. 62, Theorem 1]) (or directly from the second coefficient theorem for $f$ ) that

$$
|c|=\frac{2}{4+\left|f^{\prime \prime}(0)\right|} \geqq \frac{1}{4} \text {. }
$$

In case $|c|=1 / 4$ we have $\left|f^{\prime \prime}(0)\right|=4$, so that $f=k_{\alpha}$ for a suitable $\alpha$. Hence (3.3) with $R=0$, or $R=4$ because $k_{\alpha}=k_{-\alpha} /\left\{1+4(-\alpha) k_{-\alpha}\right\}$. In case $|c|>1 / 4$, we consider $g$ of (3.4) for the present $c$. Since the equalities in (3.5) hold, it follows that $g=k_{\alpha}$ for a suitable $\alpha$. Consequently,

$$
f=\frac{c k_{\alpha}}{c+k_{\alpha}} \text {. }
$$

Since $f$ is pole-free, $k_{\alpha}$ must omit the value $-c$ in $D$. Hence

and finally (3.3).

$$
1 / c=\alpha R, \quad 0<R<4,
$$

Proof of Theorem 1. Set $\rho=\rho(z, f)(>0)$ and consider $g$ of (2.3). Apply Lemma 3.1 to $g \in S$ to obtain

$$
\frac{2}{4+2 \rho \gamma(z, f)} \leqq \delta(0, g) \text {. }
$$

The estimate (3.1) now follows from (3.6), combined with

$$
\delta(z, f) \geqq \rho F(z, f) \delta(0, g) .
$$

For $f$ of the form (1.2) we have $\rho(z, f)=1, F(z, f)=|p|, \gamma(z, f)=|2-R|$, and

$$
\delta(z, f)= \begin{cases}\frac{|p|}{4-R} & \text { if } R \leqq 2 ; \\ \frac{|p|}{R} & \text { if } R>2 .\end{cases}
$$

It is now easy to observe that the equality holds in (3.1). Conversely suppose 
that the equality holds in (3.1). Then the equality in (3.6) holds. It then follows from Lemma 3.1 that $g$ is of the form (3.3). Setting $p=\rho\left(1-|z|^{2}\right) f^{\prime}(z)$ and $q=f(z)$ we now have

$$
f(w)=\frac{p k_{\alpha}\left(\frac{\rho^{-1}(w-z)}{1-\bar{z} w}\right)}{1+R \alpha k_{\alpha}\left(\frac{\rho^{-1}(w-z)}{1-\bar{z} w}\right)}+q, \quad w \in D .
$$

If $\rho<1$, then $f$ must have a pole at $w_{0} \in D$, where $w_{0}=\left(\rho \eta_{0}+z\right) /\left(1+\bar{z} \rho \eta_{0}\right)$ with $k_{\alpha}\left(\eta_{0}\right)=-1 /(R \alpha)(=\infty$ if $R=0)$ and $\left|\eta_{0}\right|=1$. This contradiction shows that $\rho=1$. This completes the proof that $f$ must be of the form (1.2).

E. Netanyahu [Nt, p. 321, Theorem 3] claimed that, for $f \in S$,

$$
\left(\frac{4+\sqrt{2(2-A)}}{6+A}\right)^{2} \leqq \delta(0, f), \quad A=\left|f^{\prime \prime}(0)\right| / 2 ;
$$

this result is also referred to in the textbook [G, II, p. 115]. However, this estimate of Netanyahu has a counter-example for each $A, 0 \leqq A<2$. Consider the special function

$$
f=\frac{k}{1+(2-A) k} \in S \quad(0 \leqq A<2) .
$$

Then, $f^{\prime \prime}(0) / 2=A$ and $\delta(0, f)=1 /(2+A)$. On the other hand, some calculations show that

$$
\frac{1}{2+A}<\left(\frac{4+\sqrt{2(2-A)}}{6+A}\right)^{2} \quad \text { for } 0 \leqq A<2 .
$$

Our Lemma 3.1 claims that for each $f \in S$,

$$
\frac{1}{2+A} \leqq \delta(0, f), \quad A=\left|f^{\prime \prime}(0)\right| / 2,
$$

and the estimate is sharp for all $A, 0 \leqq A \leqq 2$.

Remark. Combining (2.1) and (3.1) we have

$$
\frac{\rho(z, f) F(z, f)}{4} \leqq \delta(z, f)
$$

at $z$ where $f^{\prime}(z) \neq 0$. The equality holds if and only if $f$ is of the form (2.2). If $f^{\prime}(z)=0$, then both sides of (3.7) are zero. One might say that (3.7) is an improvement of (II) because $\rho(z, f) \equiv 1$ if $f$ is univalent in $D$.

4. Lower estimate I. We consider $\gamma$ and $\delta$ in

THEOREM 2. Let $f$ be holomorphic in $D$ and suppose that $f^{\prime}(z) \neq 0$ at a point $z \in D$. Then 


$$
\delta(z, f) \leqq \frac{2 F(z, f)}{2+\gamma(z, f)} .
$$

The equality holds if and only if $f$ is of the form (1.1).

Some properties of rotations $k_{\alpha, M} \in S$ of the bounded Koebe function $k_{1, M}$ are noted here $\left(k_{1, M}(z)=k(z, M)\right.$ in $\left.[\mathbf{G}, \mathrm{I}, \mathrm{p} .38]\right)$. For $M \geqq 1$ we set

$$
k_{1, M}(z)=M k^{*}(k(z) / M), \quad z \in D,
$$

where $k^{*}$ defined in $k(D)$ is the inverse of the Koebe function $k$. Furthermore, for $\alpha,|\alpha|=1$, we set

$$
k_{\alpha, M}(z)=\alpha^{-1} k_{1, M}(\alpha z), \quad z \in D .
$$

Hence $k_{\alpha, 1}(z) \equiv z$. The limit of $k_{\alpha, M}(z)$ as $z \rightarrow-\alpha^{-1}$ is denoted:

$$
k_{\alpha, M}\left(-\alpha^{-1}\right)=-\alpha^{-1} M\{2 M-1-2 \sqrt{M(M-1)}\} \text {. }
$$

The function $k_{\alpha, M}$ maps $D$ one-to-one onto $\{|z|<M\}$ slit along

$$
\left\{t \alpha^{-1} ;-M<t \leqq k_{1, M}(-1)\right\} \quad(=\emptyset \text { if } M=1) .
$$

Thus,

$$
\delta\left(0, k_{\alpha, M}\right)=-k_{1, M}(-1)=M\{2 M-1-2 \sqrt{M(M-1)}\} .
$$

We shall make use of

Lemma 4.1. ([Pi, p, 252]; see [G, I, p. 38, Theorem 4]) For $f \in S$ with $|f|<M$ in $D$ we have

$$
\left|f^{\prime \prime}(0)\right| \leqq 4\left(1-\frac{1}{M}\right)
$$

The equality in (4.2) holds if and only if $f=k_{\alpha, M}$.

Proof of Theorem 2. Set $F=F(z, f)(>0)$ and $\delta=\delta(z, f)(>0)$. Let $H$ be the inverse function of $f$ in the disk $\{w ;|w-f(z)|<\delta\}$ such that $H(f(z))=z$. Then the function

$$
g(w)=\frac{\left(1-|z|^{2}\right) f^{\prime}(z)}{\delta} \cdot \frac{H(\delta w+f(z))-z}{1-\bar{z} H(\delta w+f(z))}
$$

of $w \in D$ is in $S$ with $|g|<M \equiv F / \delta$. Since

$$
g^{\prime \prime}(0)=\frac{-2 \delta a(z, f)}{\left(1-|z|^{2}\right) f^{\prime}(z)},
$$

it follows from Lemma 4.1 for $g$ that

$$
\frac{\gamma(z, f)}{M}=\frac{\left|g^{\prime \prime}(0)\right|}{2} \leqq 2\left(1-\frac{1}{M}\right),
$$


so that

$$
\frac{1}{M} \leqq \frac{2}{2+\gamma(z, f)} .
$$

This is (4.1). If $f$ is of the form (1.1), then we have $F(z, f)=|p|, \gamma(z, f)=0$ and $\delta(z, f)=|p|$, so that the equality holds in (4.1). Conversely suppose that the equality in (4.1) holds, so that the equality in (4.4) holds. It follows from Lemma 4.1 applied to $g$ of (4.3) that $g=k_{\alpha, M}$. Then

$$
\eta \equiv \frac{(\beta M)^{-1} k_{\alpha, M}(w)+z}{1+\bar{z}(\beta M)^{-1} k_{\alpha, M}(w)}=H(\delta w+f(z)) \quad \text { for } w \in D,
$$

where $|\beta|=1, f^{\prime}(z)=\beta\left|f^{\prime}(z)\right|$. Suppose that $M>1$. Then, the mapping $\eta \rightarrow w$ is one-to-one and conformal from $D$ slit along $L$ onto $D$. Here $L$ is the part of the circle or the line, passing through $z$ and orthogonal to $\partial D$, between the points

$$
\eta_{1}=\frac{-(\alpha \beta)^{-1}+z}{1-(\alpha \beta)^{-1} \bar{z}} \quad \text { and } \quad \eta_{2}=\frac{(\alpha \beta M)^{-1} k_{1, M}(-1)+z}{1+(\alpha \beta M)^{-1} k_{1, M}(-1) \bar{z}}
$$

note that $\left|\eta_{1}\right|=1$ and $\left|\eta_{2}\right|<1$. Hence

$$
f(\eta)=\delta w+f(z), \quad \eta \in D \backslash L .
$$

But if $\eta_{0} \in L \backslash\left\{\eta_{1}, \eta_{2}\right\}$, then we have distinct points $w_{1}$ and $w_{2}$ on the circle $\partial D$ such that $w \rightarrow w$, as $\eta(\in D \backslash L) \rightarrow \eta_{0}$ from one side of $L(j=1,2)$. Accordingly, (4.5) shows that

$$
\delta w_{1}+f(z)=f\left(\eta_{0}\right)=\delta w_{2}+f(z) .
$$

This is a contradiction. We hereby have $M=1$. Consequently, $\beta^{-1} w=(\eta-z) /$ $(1-\bar{z} \eta)$, and

$$
f(\eta)=\frac{\beta \delta(\eta-z)}{1-\bar{z} \eta}+f(z), \quad \eta \in D
$$

Remark. Theorems 2 and 1 now yield $\left(\mathrm{I}^{\prime}\right)$ and (II') in Section 1 , respectively.

5. Lower estimate II. We consider $\rho$ and $\delta$ in

THEOREM 3. Let $f$ be holomorphic in D. Then

$$
\delta(z, f) \leqq \frac{4 \rho(z, f) F(z, f)}{\{\rho(z, f)+1\}^{2}}
$$

at each $z \in D$. The equality at $z$ with $f^{\prime}(z) \neq 0$ holds if and only if $f$ is of the form (1.1).

The right-hand side of $(5.1)$ is not greater than $F(z, f)$, so that (5.1) is an 
improvement of (I) in terms of $\rho(z, f)$.

LEMMA 5.1. For $f \in S$ with $|f|<M$ in $D$ we have

$$
-k_{1, M}(-1) \leqq \delta(0, f) \text {. }
$$

The equality holds in (5.2) if and only if $f=k_{\alpha, M}$.

The inequality (5.2) is due to G. Pick (see (IV') for $R=M$ and for $|z| \rightarrow 1$ in [Pi, p. 261]; see [G, II, p. 81, Problem 37] also.) The equality discussion in the present form is never given in the literatures and should be proved. The hint of Goodman [G, II, p. 287] for the cited problem appears to be not suited for the argument on the equality.

Let $c \in\{z ;|z| \leqq M\} \backslash f(D)$. We choose $\beta$ with $|\beta|=1, \beta c=-|c|$. Then

$$
g=\frac{M}{\beta} k\left(\frac{\beta f}{M}\right)=\frac{M^{2} f}{(M-\beta f)^{2}}
$$

is a member of $S$ and $g$ omits the value

$$
\frac{c}{\left(1+|c| M^{-1}\right)^{2}} \text {. }
$$

It then follows from the Koebe one-quarter theorem that $\frac{|c|}{\left(1+|c| M^{-1}\right)^{2}} \geqq \frac{1}{4}$ or

$$
|c| \geqq-k_{1, M}(-1)
$$

and the equality holds if and only if $g=k_{\alpha}$. Our requested (5.2) follows from (5.5). For the equality we first remember that $\delta\left(0, k_{\alpha, M}\right)=-k_{1, M}(-1)$. Conversely suppose that the equality in (5.2) holds. Choose $c \in \partial f(D)$ such that $|c|=\delta(0, f)\left(=-k_{1, M}(-1)\right)$. Then $g$ of $(5.3)$ for the present $c$ must be $k_{\alpha}$. The image of $D$ by $g=k_{\alpha}$ is $C$ slit along the half line $\left\{t \alpha^{-1} ; t \leqq-1 / 4\right\}$. On the other hand, the same $g$ of the form (5.3) omits the values on $\left\{t \beta^{-1} ; t \leqq-M / 4\right\}$. Hence $\alpha=\beta$. Consequently,

$$
M \alpha^{-1} k\left(\frac{\alpha f(w)}{M}\right) \equiv k_{\alpha}(w), \quad w \in D
$$

yields $f=k_{\alpha, M}$.

Proof of Theorem 3. We may suppose that $f^{\prime}(z) \neq 0$. Again, $g$ of (4.3) is in $S$ and $|g|<M$, where $M=F / \delta$. Thus Lemma 5.1, applied to $g$, yields the following chain of inequalities:

$$
-k_{1, M}(-1) / M \leqq \delta(0, g) / M \leqq \rho \equiv \rho(z, f) .
$$

The left-most is a decreasing function of $M \geqq 1$, so that we have

$$
\frac{1}{4}\left(\rho+\frac{1}{\rho}+2\right) \leqq M
$$


whence (5.1). The equality argument is similar to that in the proof of Theorem 2 , so that it is omitted.

Can the right-hand sides of (4.1) and (5.1), in case $f^{\prime}(z) \neq 0$, be comparable in the sense that one of them is always greater than the other? For the negative answer we first note that the problem is reduced to the comparison of the functions

$$
\Phi(z, f)=1 /\{2+\gamma(z, f)\} \quad \text { and } \quad \Psi(z, f)=2 \rho(z, f) /\{\rho(z, f)+1\}^{2}
$$

at $z$ where $f^{\prime}(z) \neq 0$. A counter-example is supplied by the function $f(z) \equiv z^{2}$ for which we have $\Phi(z, f)=2|z| /\left\{4|z|+\left.|1-3| z\right|^{2} \mid\right\}$ and $\Psi(z, f)=2|z| /\{|z|+1\}^{2}$ for all $z \in D \backslash\{0\}$ because $\rho(z, f)=|z|$ there. Note that $f$ is univalent in each half disk of $D$. Hence, for $f(z)=z^{2}$ we have

$$
\begin{array}{ll}
\Phi(z, f)<\Psi(z, f) & \text { if } 0<|z|<1 / 2 \text { or } \frac{\sqrt{5}-1}{2}<|z|<1 \\
\Phi(z, f)=\Psi(z, f) & \text { if }|z|=1 / 2 \text { or }|z|=\frac{\sqrt{5}-1}{2} \\
\Phi(z, f)>\Psi(z, f) & \text { if } 1 / 2<|z|<\frac{\sqrt{5}-1}{2}
\end{array}
$$

Finally, if $f$ is univalent in $D$, then we always have $\Phi(z, f) \leqq 1 / 2 \equiv \Psi(z, f)$.

Remark 1. Seidel and Walsh [SW, p. 134] pointed out that the equality in ( I ) holds if $f(w)=(w-z) /(1-\bar{z} w)$. We can easily prove that, under the condition $f^{\prime}(z) \neq 0$, the equality holds if and only if $f$ is of the form (1.1). It suffices to consider the case where the modulus of the derivative at $w=0$ of the following function of $w$ :

$$
\frac{H(\delta w+f(z))-z}{1-\bar{z} H(\boldsymbol{\delta} w+f(z))}
$$

is one, where $\delta=\delta(z, f)>0$.

The situation is the same for the estimate in (II) under the condition $f^{\prime}(z)$ $\neq 0$. This is obtained by applying the Koebe one-quarter theorem to

$$
\frac{f\left(\frac{w+z}{1+\bar{z} w}\right)-f(z)}{\left(1-|z|^{2}\right) f^{\prime}(z)} \in S .
$$

The equality holds if and only if this function is $k_{\alpha}(w)$, so that we immediately observe that $f$ must be of the form (1.2) with $R=0$. The equality discussion in $[\mathbf{M c}, \mathrm{pp} .10-11]$ is incomplete.

Remark 2. The function $\delta(z, f) / \rho(z, f)$ for nonconstant $f$ is continuous in $D$ except for the zeros of $f^{\prime}$. It follows from (3.1), (2.1), and (5.1) that

$$
(1 / 4) \delta(z, f) / \rho(z, f) \leqq F(z, f) \leqq 4 \delta(z, f) / \rho(z, f)
$$


at each $z \in D$ with $f^{\prime}(z) \neq 0$. Since $F(z, f)$ is continuous in $D$ and is zero if and only if $f^{\prime}(z)=0$, the same is true of $\delta(z, f) / \rho(z, f)$ if we define this to be zero at the zeros of $f^{\prime}$. An application is that a nonconstant holomorphic function $f$ in $D$ is Bloch, that is, $F(z, f)$ is bounded in $D$, if and only if $\delta(z, f) / \rho(z, f)$ is bounded in $D$. The quantity $\delta(z, f)$ is "Euclidean", while $\rho(z, f)$ is "nonEuclidean". Thus, it would be of interest to compare these with

$$
F(z, f)=\lim _{w \rightarrow z} \frac{|f(w)-f(z)|}{\{|z-w| /|1-\bar{z} w|\}} .
$$

The numerator in the quotient in the right is "Euclidean", while the denominator is "non-Euclidean".

6. The Schwarzian derivative. Let

$$
\sigma(f)=\frac{f^{\prime \prime \prime}}{f^{\prime}}-\frac{3}{2}\left(\frac{f^{\prime \prime}}{f^{\prime}}\right)^{2}
$$

be the Schwarzian derivative of $f$ nonconstant and meromorphic in $D$. For the later consideration of the Poincaré density we prove two upper estimates of the Nehari derivative:

$$
N(z, f)=\frac{1}{2}\left(1-|z|^{2}\right)^{2}|\sigma(f)(z)|
$$

of $f$ at $z \in D$. The reason why we call $N(z, f)$ the Nehari derivative is obvious; $Z$. Nehari $\left[\mathbf{N h}\right.$ ] proved that if $\sup _{z \in D} N(z, f) \leqq 1$, then $f$ is univalent in $D$ and $\mathrm{E}$. Hille [Hi] proved the sharpness of the constant one. To make our style consistent with the propositions up to now, we restrict ourselves within the pole-free case; the possible extensions to the meromorphic case will be left as exercises.

THEOREM 4. Let $f$ be holomorphic in $D$ and suppose that $f^{\prime}(z) \neq 0$ at $z \in D$. Then we have the following two propositions.

(i) We have

$$
N(z, f) \leqq \frac{3}{\rho(z, f)^{2}} .
$$

The equality holds if and only if $f$ is of the form (1.2).

(ii) We have

$$
N(z, f) \leqq 3\left(\left\{\frac{F(z, f)}{\delta(z, f)}\right\}^{2}-1\right) .
$$

The equality holds if and only if $f$ is of the form (1.1).

For $M \geqq 1$ and for $0 \leqq R \leqq 4\left(1-\frac{1}{M}\right)$ we set 


$$
k_{1, M, R}=M k^{*}\left(\frac{k / M}{1+R k}\right), \quad k_{\alpha, M, R}(z)=\alpha^{-1} k_{1, M, R}(\alpha z), \quad|\alpha|=1, z \in D .
$$

Then, $k_{\alpha, M, R} \in S$ and $|f|<M$ in $D$. Further, $k_{\alpha, M}=k_{\alpha, M, 0}$, so that $k_{\alpha, 1,0}(z) \equiv z$.

LEMma 6.1. If $f \in S$, then $|\sigma(f)(0)| \leqq 6$. The equality holds if and only if $f$ is of the form (3.3). If $f \in S$ and $|f|<M$ in $D$, then we have

$$
|\sigma(f)(0)| \leqq 6\left(1-\frac{1}{M^{2}}\right) .
$$

The equality in (6.3) holds if and only if $f=k_{\alpha, M, R}$.

The first half is due to L. Bieberbach; see $[\mathbf{G}, I$, p. 35, Theorem 2]. Actually, if

$$
f(z)=z+a_{2} z^{2}+a_{3} z^{3}+\cdots,
$$

then $\sigma(f)(0)=6\left(a_{3}-a_{2}^{2}\right)$. For the equality, Goodman [G, I, p. 35] adopted the parameter $\phi, 0 \leqq \phi<2 \pi$, such that $2 \cos \phi=2-R$ for $R$ in (3.3). The second half is in $[\mathbf{G}, \mathbf{I}$, p. 46 , Problem 13$]$. To be more complete we give here a detailed explanation on the equality.

There always exists $\beta$ such that $|\beta|=1$ and $\sigma(f)(0) \beta^{2}=-|\sigma(f)(0)|$. Set

$$
g(z)=M k\left(\frac{\beta^{-1} f(\beta z)}{M}\right), \quad z \in D .
$$

Then it follows from the first half of Lemma 6.1, applied to $g \in S$, together with $\sigma(g)(0)=-6 / M^{2}-|\sigma(f)(0)|$, that $6 / M^{2}+|\sigma(f)(0)| \leqq 6$; this is (6.3). It is easy to observe that

$$
\left|\sigma\left(k_{\alpha, M, R}\right)(0)\right|=6\left(1-\frac{1}{M^{2}}\right) .
$$

Conversely we suppose that the equality in (6.3) holds. Then $|\sigma(g)(0)|=6$, so that $g$ must be of the form (3.3) or $g=k_{\alpha} /\left(1+R \alpha k_{\alpha}\right)$. Now the image of $D$ by $g=k_{\alpha} /\left(1+R \alpha k_{\alpha}\right)$ is $C$ slit along $\left\{t \alpha^{-1} ; 1 / R \leqq t\right\} \quad(=\emptyset$ if $R=0)$ and $\left\{t \alpha^{-1} ; t \leqq\right.$ $1 /(R-4)\}$ (= $\emptyset$ if $R=4$ ). On the other hand the same $g$ of the form (6.4) must omit at least the values on $\{t ; t \leqq-M / 4\}$. Hence the possible cases are two: $\alpha=1(R<4)$ and $\alpha=-1(R>0)$. In case $\alpha=1$, it follows from $-M / 4 \leqq 1 /(R-4)$ or $R \leqq 4(1-(1 / M))$ that $f=k_{(1 / \beta), M, R}$. In case $\alpha=-1$, the similar procedure yields that

$$
f(z)=\beta M k^{*}\left(\frac{-k\left(-\beta^{-1} z\right) / M}{1+R k\left(-\beta^{-1} z\right)}\right), \quad z \in D,
$$

where $4 \geqq R \geqq 4 / M$. Since $k(-\eta)=-k(\eta) /(4 k(\eta)+1), \eta \in D$, it follows that $f=$ $k_{(1 / \beta), M, 4-R}$, or

$$
f=k_{(1 / \beta), M, Q}, \quad 0 \leqq Q \leqq 4\left(1-\frac{1}{M}\right)
$$


This is again the requested form.

Proof of Theorem 4. The first half. To $g \in S$ of (2.3) we apply the first half of Lemma 6.1. Then

$$
\rho^{2}\left(1-|z|^{2}\right)^{2}|\sigma(f)(z)|=|\sigma(g)(0)| \leqq 6 .
$$

This is (6.1). The equality in the last of (6.5) holds if and only if $g$ is of the form (3.3). By the same reasoning as in the proof of Theorem 1 we have $\rho=1$, whence $f$ is of the form (1.2). The second half. For $g \in S$ of (4.3) we apply the second half of Lemma 6.1. Then

$$
\frac{2 \delta^{2}}{F^{2}} N(z, f)=\frac{\delta^{2}|\sigma(f)(z)|}{\left|f^{\prime}(z)\right|^{2}}=|\sigma(g)(0)| \leqq 6\left(1-\frac{1}{M^{2}}\right),
$$

where $1 / M=\delta / F$. This is (6.2). The equality in the right-most in (6.6) holds if and only if $g=k_{\alpha, M, R}$. The equality in (6.2) holds for each $f$ of the form (1.1). Conversely suppose that the equality in (6.2) holds. The rest of the proof is similar to that of Theorem 2 . If $M>1$, then

$$
\eta \equiv \frac{(\beta M)^{-1} k_{\alpha, M, R}(w)+z}{1+\bar{z}(\beta M)^{-1} k_{\alpha, M, R}(w)}=H(\delta w+f(z)) \quad \text { for } w \in D,
$$

and $\eta \rightarrow w$ represents a one-to-one conformal mapping from $D$ slit along at least one and at most two simple curves, onto $D$. We arrive at the contradiction by the same argument. Hence $M=1$ and consequently $R=0$. Thus $f$ must be of the form (1.1).

The right-hand sides of (6.1) and (6.2) which we denote by $\Phi^{*}(z, f)$ and $\Psi^{*}(z, f)$, respectively, cannot again be comparable. For the same function $f(z)=z^{2}$ considered in Section 5 we have $\rho(z, f)=|z|$ again and $F(z, f)=$ $2|z|\left(1-|z|^{2}\right)$ in $D \backslash\{0\}$ and further,

$$
\delta(z, f)= \begin{cases}|z|^{2} & \text { if } 0<|z|^{2} \leqq 1 / 2 \\ 1-|z|^{2} & \text { if }|z|^{2}>1 / 2\end{cases}
$$

Elementary but tedious calculations show that

$$
\begin{aligned}
& \Phi^{*}(z, f)<\Psi^{*}(z, f) \quad \text { if } 0<|z|^{2}<\frac{9-\sqrt{33}}{8} \text { or } \frac{\sqrt{17}+1}{8}<|z|^{2}<1 ; \\
& \Phi^{*}(z, f)=\Psi^{*}(z, f) \quad \text { if }|z|^{2}=\frac{9-\sqrt{33}}{8} \text { or }|z|^{2}=\frac{\sqrt{17}+1}{8} \\
& \Phi^{*}(z, f)>\Psi^{*}(z, f) \quad \text { if } \frac{9-\sqrt{ } 3 \overline{3}}{8}<|z|^{2}<\frac{\sqrt{17}+1}{8} .
\end{aligned}
$$

7. The Poincaré density. We return to $G$ with the Poincare density $P_{G}$ as described in Section 1. We set $\rho_{G}(z)=\rho(w, f)$ for $z \in G$, where $f \in \operatorname{Proj}(D, G)$ 
and $z=f(w)$. It is not difficult to prove that $\rho(w, f)$ is independent of the particular choice of $f$ and $w$ as far as $z=f(w)$. We apply (2.1), (3.1), (4.1), and (5.1) to $f \in \operatorname{Proj}(D, G)$; we note that $f^{\prime}$ never vanishes in $D$. The results obtained are the following (7.1)-(7.4).

$$
\rho_{G}(z) \gamma_{G}(z) \leqq 2, \quad z \in G .
$$

The equality holds at (only) one point $z \in G$ if and only if $G$ is $C$ slit along a half line whose extension contains $z$.

$$
\frac{\rho_{G}(z)}{2+\rho_{G}(z) \gamma_{G}(z)} \leqq \delta_{G}(z) P_{G}(z), \quad z \in G .
$$

The equality holds at (only) one point $z \in G$ if and only if $G$ is $C$ slit along the half lines (1.3) and (1.4) and $z=q$.

$$
\delta_{G}(z) P_{G}(z) \leqq \frac{2}{2+\gamma_{G}(z)}, \quad z \in G .
$$

The equality holds at (only) one point $z \in G$ if and only if $G$ is a disk and $z$ is its center. In particular, $\delta_{G}(z) P_{G}(z)<1$ everywhere in $G$ except possibly for one point $z_{0}$ if and only if $G$ is a disk of center $z_{0}$.

$$
\delta_{G}(z) P_{G}(z) \leqq \frac{4 \rho_{G}(z)}{\left(\rho_{G}(z)+1\right)^{2}}, \quad z \in G .
$$

The equality holds at (only) one point $z \in G$ if and only if $G$ is a disk and $z$ is its center.

We set

$$
\begin{aligned}
& A(G)=\inf _{z \in G} \delta_{G}(z) P_{G}(z), \\
& \gamma(G)=\sup _{z \in G} \gamma_{G}(z), \\
& \rho(G)=\inf _{z \in G} \rho_{G}(z) .
\end{aligned}
$$

We call $G$ of finite type if $A(G)>0$. In general, $A(G) \leqq 1$ by ( I ) and if $G$ is simply connected, then $A(G) \geqq 1 / 4$ by (II).

First, $\gamma(G) \leqq 2 / \rho(G)$ by (7.1). We have from (7.3) and (7.4) that

$$
A(G) \leqq\left\{\begin{array}{l}
\frac{2}{2+\gamma(G)} \\
\frac{4 \rho(G)}{(\rho(G)+1)^{2}} .
\end{array}\right.
$$

On the other hand, it follows from C. Pommerenke's result [Po, p. 115, (1.11) or p. 116, Lemma 1.3] (see [G, II, p. 114]), applied to a projection, that $A(G) \geqq$ $1 /\{2 \gamma(G)\}$, which, together with $\gamma(G) \leqq 2 / \rho(G)$, shows that

$$
\rho(G) / 4 \leqq 1 /\{2 \gamma(G)\} \leqq A(G) .
$$


It further follows from Pommerenke's another result [Po, p. 134, Folgerung 2.5] (see [G, II, p. 110]) that $\rho(G) \geqq 1 / \gamma(G)$. Hence $1 \leqq 1 / \rho(G) \leqq \gamma(G) \leqq 2 / \rho(G)$. Consequently, $G$ is of finite type if and only if $\gamma(G)<+\infty$ if and only if $\rho(G)>0$. We note that

$$
\frac{\rho(G)}{2+\rho(G) r(G)} \leqq A(G)
$$

resulting from (7.2) is not good since the left-hand side is not greater than $1 /\{2 \gamma(G)\}$. However, this again yields $\rho(G) / 4 \leqq A(G)$.

Since $\gamma(G) \geqq 1$ it follows from (7.5) that $A(G) \leqq 2 / 3$ for general $G$. If $A(G)$ $=2 / 3$, then (7.5) yields that $\gamma(G) \leqq 1$, hence $\gamma(G)=1$. Thus, $G$ must be convex (see [Po, p. 134, Folgerung 2.4]). We consequently have the problem of finding the best possible, absolute constant $c>0$ such that $A(G) \geqq c$ implies that $G$ is simply connected. More generally, we have the inverse problem to (II): Find the smallest of the absolute constants $c>0$ such that the condition $\inf _{z \in D}\{\delta(z, f) /$ $F(z, f)\} \geqq c$ for $f$ holomorphic with nonvanishing $f^{\prime}$ in $D$, implies the univalency of $f$ in $D$. We know that the smallest is not greater than $2 / 3$.

We next set

$$
B(G)=\sup _{z \in G}\left|\left(\partial^{2} / \partial z^{2}\right)\left\{1 / P_{G}(z)\right\}\right| / P_{G}(z) .
$$

Then $G$ is of finite type if and only if $B(G)<+\infty$. This follows from

$$
\min (1,1 / B(G)) \leqq \rho(G)^{2} \leqq 3 / B(G) .
$$

For the proof of (7.6) we need

LEMMA 7.1. Let $f$ be holomorphic with nonvanishing $f^{\prime}$ in $D$. Then

$$
\min (1,1 /\|f\|) \leqq \inf _{z \in D} \rho(z, f)^{2},
$$

where

$$
\|f\|=\sup _{z \in D} N(z, f)
$$

Proof. If $\|f\| \leqq 1$, then the cited Nehari theorem reads $\rho(z, f) \equiv 1$ in $D$. Suppose that $1<\|f\|$. If $\|f\|=+\infty$, then we have nothing to prove. Suppose that $1<\|f\|<+\infty$ and set $r=1 / \sqrt{\|f\|}$. For $z \in D$ we set $\varphi(w)=(r w+z) /(1+\bar{z} r w)$, $w \in D$. Then

$$
\left(1-|w|^{2}\right)\left|\varphi^{\prime}(w)\right| \leqq r\left(1-|\varphi(w)|^{2}\right), \quad w \in D .
$$

This, together with

$$
\sigma(g)(w)=\sigma(f)(\varphi(w)) \varphi^{\prime}(w)^{2}, \quad w \in D,
$$

for $g=f \circ \varphi$, shows that $\|g\| \leqq r^{2}\|f\|=1$, whence $g$ is univalent in $D$ and

$$
1 /\|f\|=r^{2} \leqq \rho(z, f)^{2} .
$$


We remember that

$$
\left|\left(\partial^{2} / \partial z^{2}\right)\left\{1 / P_{G}(z)\right\}\right| / P_{G}(z)=N(w, f)
$$

for $f \in \operatorname{Proj}(D, G), z=f(w), w \in D$; see [Y, p. 168, (3.3)].

The first half of (7.6) is then a consequence of (7.7). The right-hand side of (7.6) is a consequence of (6.1) applied to $f \in \operatorname{Proj}(D, G)$, that is,

$$
\left|\left(\partial^{2} / \partial z^{2}\right)\left\{1 / P_{G}(z)\right\}\right| / P_{G}(z) \leqq 3 / \rho_{G}(z)^{2}, \quad z \in G .
$$

It is now easy to obtain the following from (6.2):

$$
B(G) \leqq 3\left(\frac{1}{A(G)^{2}}-1\right) \text {. }
$$

The results in the present section and those in $[\mathbf{Y}$, Theorem 1 and Section 6] thus compensate for each other.

8. Further about domains of finite type. We continue the study of $P_{G}$. A.F. Beardon and Pommerenke $[\mathbf{B P}]$ considered the quantity

$$
\beta_{G}(z)=\inf \left\{\left|\log \frac{\delta_{G}(z)}{|a-b|}\right| ; a, b \in \partial G \text { and }|z-a|=\delta_{G}(z)\right\}
$$

for $z \in G$. Actually, the infimum is attained by a pair $a, b$ on $\partial G$. In the light of J.A. Hempel's sharp form of the Landau inequality (8.2) below, Beardon and Pommerenke's theorem [BP, Theorem 1] should be

THEOREM 5 [BP]. At each point $z \in G$ we have

$$
\frac{1}{2\left\{\beta_{G}(z)+c_{H}\right\}} \leqq \delta_{G}(z) P_{G}(z) \leqq \frac{\pi}{4 \beta_{G}(z)},
$$

where $c_{H}=\frac{\Gamma(1 / 4)^{4}}{4 \pi^{2}}=4.376 \cdots$ is Hempel's constant.

The proof of the left-hand side of (8.1) is the same as in [BP] except for the use of the Hempel estimate $[\mathrm{He}$, p. $443,(4.1)]$ for $P^{*}=P_{C \backslash(0,11}$, namely,

$$
1 / P^{*}(z) \leqq 2|z|\left\{|\log | z||+c_{H}\right\}, \quad z \in C \backslash\{0,1\},
$$

where $c_{H}=\left\{2 P^{*}(-1)\right\}^{-1}$; note that Hempel adopted the Poincaré density $2 P_{G}$. The equality in (8.2) holds at $z=-1$. If $G=C \backslash\{0,1\}$, then the equality holds at $z=-1$ in the left-hand side of (8.1). If $G$ is simply connected, then one can easily show that $\beta_{G}(z) \equiv 0$, so that the right-hand side of $(8.1)$ is trivial.

It follows from Theorem 5 that $G$ is of finite type if and only if

$$
\beta(G) \equiv \sup _{z \in G} \beta_{G}(z)
$$


is finite. More precisely, (8.1) yields

$$
\frac{1}{2\left\{\beta(G)+c_{H}\right\}} \leqq A(G) \leqq \frac{\pi}{4 \beta(G)} .
$$

If $G$ is simply connected, then $\beta(G)=0$, so that $A(G) \geqq 1 /\left\{2 c_{H}\right\}$, a worse result than $A(G) \geqq 1 / 4$.

For the later use we slightly change the formulation of Beardon and Pommerenke's Corollary 1 , together with their proof, in $[\mathbf{B P}]$ in

Proposition [BP]. A domain $G$ is of finite type if and only if there exists a constant $K \equiv K(G)>1$ such that, if a closed disk $\{|z-a| \leqq r\} \quad(a \in C, r>0)$ meets $\partial G$, then either no ring domain $\{r<|z-a|<R\}$ is contained in $G$, or else $R \leqq K r$ whenever $\{r<|z-a|<R\} \subset G$.

To prove that $K=\exp \{\pi / A(G)\}$ will do if $A(G)>0$, we suppose that $\{|z-a| \leqq r\}$ meets $\partial G$ and

$$
Q \equiv\{r<|z-a|<R\} \subset G .
$$

Then, $r=q e^{-m}$ and $R=q e^{m}$ for $q=\sqrt{r R}$ and $m=(1 / 2) \log (R / r)$. Since $w=a+q$ $\in Q$, it follows that

$$
\delta_{G}(w) \leqq q+r, \quad P_{G}(w) \leqq P_{Q}(w)=\frac{\pi}{4 m q} ;
$$

actually, $w=f(0)$ and $P_{Q}(w)=1 /\left|f^{\prime}(0)\right|$, where

$$
f(z)=a+q e^{-m} \exp \left\{\frac{-2 m i}{\pi} \log \frac{1+z}{1-z}+m\right\} \in \operatorname{Proj}(D, Q) .
$$

Hence

$$
A(G) \leqq \delta_{G}(w) P_{G}(w) \leqq \frac{\pi}{4 m}\left(1+e^{-m}\right)<\frac{\pi}{2 m}
$$

shows that $R \leqq r K$. Conversely we shall prove that $\beta(G) \leqq(1 / 2) \log K$ if $K$ exists. Suppose that $\beta \equiv \beta_{G}(z)>0, z \in G$, and choose $a \in \partial G$ such that $|z-a|=\delta_{G}(z) \equiv \delta$. Then the ring domain

$$
U=\left\{w \in C ;\left|\log \frac{\delta}{|w-a|}\right|<\beta\right\}=\{r<|w-a|<R\},
$$

where $r=\delta e^{-\beta}$ and $R=\delta e^{\beta}$, contains $z$, yet does not meet $\partial G$ by the definition of $\beta_{G}(z)$. Hence $U \subset G$, so that $e^{2 \beta}=R / r \leqq K$ yields $\beta_{G}(z)=(1 / 2) \log K$.

As an application of the Beardon-Pommerenke proposition above, we consider here a sufficient condition for a multiply-connected $G$ to be of finite type. Suppose that all the compact, connected components of $C \backslash G$ are at most countable and let them be $A_{1}, A_{2}, \cdots, A_{n}, \cdots$. If they are finite in number, $n_{0}$, say, then we set $A_{n}=A_{n_{0}}$ for all $n \geqq n_{0}$. Thus the components of the set 


$$
B=C \backslash\left\{G \cup\left(\bigcup_{n \geq 1} A_{n}\right)\right\}
$$

are all noncompact if $B \neq \emptyset$. Let $p(G)$ be the infimum of the diameters of $A_{n}$, $n \geqq 1$. Let $\operatorname{dis}\left(j_{1}, \cdots, j_{\nu}\right)$ be the distance of $\bigcup_{\mu=1}^{\nu} A_{\jmath_{\mu}}$ and the set

$$
C \backslash\left\{G \cup\left(\bigcup_{\mu=1}^{\nu} A_{\jmath_{\mu}}\right)\right\} \text {, }
$$

where $\nu$ is arbitrary and $j_{1}, \cdots, j_{\nu}$ are arbitrary distinct numbers. If the set of (8.3) is empty, then we define $\operatorname{dis}\left(j_{1}, \cdots, j_{\nu}\right)=+\infty$. Let $q(G)$ be the supremum of all $\operatorname{dis}\left(j_{1}, \cdots, j_{\nu}\right)$. We now have the following:

Suppose that $A_{n}$ 's cluster nowhere in $\boldsymbol{C}$ in the sense that, for each pair $a \in \boldsymbol{C}$, $r>0$, there are at most a finite number of $A_{n}$ 's which are contained in the disk $\{|z-a| \leqq r\}$. Suppose that $p(G)>0$ and $q(G)<+\infty$. Then $G$ is of finite type. More precisely,

$$
\beta(G) \leqq \frac{1}{2} \log \left\{\frac{2 q(G)}{p(G)}+1\right\} .
$$

If $A_{n}$ 's are finite in number and are nondegenerate continua, and further $B \neq \emptyset$, then $p(G)>0$ and $q(G)<+\infty$ automatically follow. Thus, M. Masumoto's lemma [Ms, Lemma 2] immediately follows. In fact we try to extend Masumoto's lemma to infinitely connected domains with some detailed constant.

For the proof of our criterion, we let $\Delta \equiv\{|z-a| \leqq r\}(a \in C, r>0)$ meet $\partial G$. If $\Delta \cap B \neq \emptyset$, then no ring domain $Q=\{r<|z-a|<R\}$ is contained in $G$. If $\Delta \cap B=\emptyset$, and if $Q \subset G$, then we let $A_{\jmath_{1}}, \cdots, A_{\nu_{\nu}}$ be all the $A_{n}$ 's contained in $\Delta$. Then, $p(G) \leqq 2 r$ and $R-r \leqq q(G)$, so that $R / r \leqq 1+2 q(G) / p(G)$. Thus we may adopt

$$
K(G)=\frac{2 q(G)}{p(G)}+1
$$

in the Beardon-Pommerenke proposition.

It is now easy to give an example of $G$ of finite type with $B=\emptyset$ and with infinitely many $A_{n}$ 's. Let $A_{n}=\left[a_{n}, b_{n}\right]$ be closed intervals on the real axis such that $a_{n} \rightarrow+\infty$ increasingly and $0<a_{n+1}-b_{n} \leqq c\left(b_{n}-a_{n}\right), n=1,2, \cdots$, where $c>0$ is a constant. Then $G=C \backslash\left(\bigcup_{n \geq 1} A_{n}\right)$ is of finite type. We may choose $K(G)=$ $2 c+1$ for the Beardon-Pommerenke criterion. For the proof we let $\Delta \equiv\{|z-a|$ $\leqq r\}$ meet $\partial G$, and suppose that $\{r<|z-a|<R\} \subset G$. Let $n$ be the largest number of $j$ such that $A_{j} \subset \Delta$. Then, $2 r \geqq b_{n}-a_{n}$ and $R-r \leqq a_{n+1}-b_{n}$, so that $R / r \leqq 1+2 c$. We consider here three specified cases where $K(G)=3$ will do.

(i) $a_{n}=2 n-2$ and $b_{n}=a_{n}+1$. In this case $p(G)=1$ and $q(G)=1$.

(ii) $a_{n}=n^{2}-n$ and $b_{n}=a_{n}+n$. In this case $p(G)=1$ and $q(G)=+\infty$.

(iii) $a_{n}=\sum_{j=1}^{n-1} \frac{j+1}{j^{2}}(=0$ if $n=1)$ and $b_{n}=a_{n}+1 / n$. In this case $p(G)=0$ and 
$q(G)=1$.

\section{REFERENCES}

[BP] A.F. Beardon and C. Pommerenke, The Poincaré metric of plane domains. J. London Math. Soc. (2) 18 (1978), 475-483.

[F] W. FENChEL, Bemerkungen über die im Einheitskreis meromorphen schlichten Funktionen. Sitzsber. Preuss. Akad. Wiss. Phys.-Math. K1. H22/23 (1931), 431436.

[G] A.W. Goodman, Univalent functions. I, II. Mariner Publ., Tampa, 1983.

[He] J.A. Hempel, The Poincaré metric on the twice punctured plane and the theorems of Landau and Schottky, J. London Math. Soc. (2) 20 (1979), 435-445.

[Hi] E. Hille, Remarks on a paper by Zeev Nehari. Bull. Amer. Math. Soc. 55 (1949), $552-553$.

[K] I. KRA, Automorphic forms and Kleinian groups. W.A. Benjamın, Reading, 1972.

[Mc] A. J. Macintrre, Two theorems on "schlicht" functions. J. London Math. Soc. 11 (1936), 7-11.

[Ms] M. MASUMOTO, A distortion theorem for conformal mappings wath an application to subharmonic functions. Hiroshima Math. J. 20 (1990), 341-350.

[Nh] Z. Nehari, The Schwarzian derivative and schlicht functions. Bull. Amer. Math. Soc. 55 (1949), 545-551.

[Nt] E. NetANYAHU, On univalent functions in the unit disk whose image contains a given disk. J. Analyse Math. 23 (1970), 305-322.

[Pi] G. PIck, Ueber die konforme Abbildung eines Kreises auf ein schlichtes und zugleich beschränktes Gebiet. Sitzsber. Kaiserl. Akad. Wiss. Wien, Math.Naturwiss. Kl. Abt. Ila 126 (1917), 247-263.

[Po] C. Pommerenke, Linear-invariante Familien analytischer Funktionen. I, Math. Ann. 155 (1964), 108-154.

[PS] G. Pólya ANd G. Szego, Problems and theorems in analysis, II. Springer-Verlag, Berlin, 1976.

[SW] W. Seidel And J. L. Walsh, On the derivatives of functions analytic in the unit circle and their radii of univalence and of $p$-valence. Trans. Amer. Math. Soc. 52 (1942), 128-216.

[Y] S. Yamashita, Univalent analytic functions and the Poincaré metric. Kodai Math. J. 13 (1990), 164-175.

Department of Mathematics

Tokyo Metropolitan University

Minami Osawa, Hachioji, Tokyo 192-03

JAPAN 